\title{
A Sort of New Improved Algorithm For Total Least Square
}

\author{
Deng Yonghe ${ }^{1,2,3, *}$
}

${ }^{1}$ College of Engineering and Designing, Lishui College, Lishui, Zhejiang, 323000, P.R. China; ${ }^{2}$ School of Geodesy and Geomatics, Wuhan University, Wuhan, 430079, P.R. China; ${ }^{3}$ School of Continuous Education, Wuhan University, Wuhan, 430079, P.R. China

\begin{abstract}
Aim to blemish of total least square algorithm based on error equation of virtual observation, this paper put forward and deduced a sort of new improved algorithm which selects essential unknown parameters among designing matrix, and then, doesn't consider condition equation of unknown parameters among designing matrix. So, this paper perfected and enriched algorithm, and sometimes, new method of this paper is better. Finally, the results of examples showed that new mothod is viable and valid.
\end{abstract}

Keywords: Error equation, essential unknown parameters, mean square error of unit weight, total least square, virtual observation.

\section{INTRODUCTION}

Literatures $[1,2]$ made some reasonable improvement such as putting forward virtual observation method and listing error equations of virtual observation for total least square algorithm, but there existed some problem. Aim to blemish of total least square algorithm based on literatures [1, 2], literatures [3] has put forward and deduced further improved algorithm which considered condition equation of unknown parameters among designing matrix. But some times, the improved algorithm isn't always better, so, this paper proposed and deduced a sort of new algorithm which selects essential unknown parameters among designing matrix, and then, doesn't consider condition equation of unknown parameters among designing matrix, perfects and enriches algorithm of total least square algorithm. Finally, imitative example showed that new method is viable and valid.

\section{THE PRINCIPLE OF IMPROVED ALGORITHM OF TOTAL LEAST SQUARE}

\subsection{Error Equations of Actual Observations}

We suppose there exists function model of adjustment of indirect observations based on actual observation [1-3]

$$
\hat{L}=\hat{B, 1} \underset{n, t}{\hat{X}}-\underset{n, 1}{d} \quad, \quad P_{n, n} L
$$

where,

$$
\hat{L}_{n, 1}=\left[\begin{array}{c}
\hat{L}_{1} \\
\hat{L}_{2} \\
\vdots \\
\hat{L}_{n}
\end{array}\right], \underset{n, t}{\hat{B}}=\left[\begin{array}{cccc}
\hat{B}_{11} & \hat{B}_{12} & \cdots & \hat{B}_{1 t} \\
\hat{B}_{21} & \hat{B}_{22} & \cdots & \hat{B}_{2 t} \\
\vdots & \vdots & \vdots & \vdots \\
\hat{B}_{n 1} & \hat{B}_{n 2} & \cdots & \hat{B}_{n t}
\end{array}\right] \text {, }
$$

*Address correspondence to this author at the College of Engineering and Designing, Lishui University, Lishui, Zhejiang, 323000, P.R. China; Tel: +86 18969588403; +8615925722009

E-mails: 1sxydengyonghe@sina.com; a15925722009@163.com

$$
\hat{X}_{t, 1}=\left[\begin{array}{c}
\hat{X}_{1} \\
\hat{X}_{2} \\
\vdots \\
\hat{X}_{t}
\end{array}\right], \underset{n, 1}{d}=\left[\begin{array}{c}
d_{1} \\
d_{2} \\
\vdots \\
d_{n}
\end{array}\right]
$$

$n$--- quantity of actual observations;

$t$--- necessary quantity of unknown parameters;

$\hat{L}_{n, 1}$--- estimated value of column vector of observations;

$\hat{B}$--- estimated value of designing matrix( part of $\hat{B}$ may $n, t$

be constant and haven't error);

$\hat{X}$---estimated value of column vector of unknown parame$t, 1$

ters;

$d$--- column vector of constant;

$n, 1$

$P L^{---}$weight matrix of actual observations.

$n, n$

We suppose any element of $\hat{B}$ can be expressed by unknown parameters

$\hat{Y}=\left(\hat{Y}_{1}, \hat{Y}_{2}, \cdots, \hat{Y}_{t^{\prime}}\right)^{T}$.

There, $\hat{Y}_{i}$ and $\hat{Y}_{j}\left(1 \leq i, j \leq t^{\prime}\right.$ and $\left.i \neq j\right)$ are independent.Then,based on Eq.1,we can obtain

$$
V_{n, 1}=\left[\begin{array}{cc}
B^{0} & A \\
n, t & n, t^{\prime}
\end{array}\right]\left[\begin{array}{c}
\hat{x} \\
t, 1 \\
\hat{y} \\
t^{\prime}, 1
\end{array}\right]-\underset{n, 1 \quad n, 1}{\left(d+B^{0} X^{0}\right)} \quad, \quad P_{t, 1} L
$$


where:

$L$---actual observations;

$n, 1$

$\underset{n, 1}{V} L^{--- \text {residuals of observations }} \underset{n, 1}{L}$;

$\hat{L}=V_{L}+L$

$n, 1 \quad n, 1 \quad n, 1$

$B^{0}$--- approximations of $\hat{B}$;

$n, t \quad n, t$

$\begin{array}{ll}\Delta \hat{B}^{---} \text {residuals of } & B^{0 ;} \\ n, t\end{array}$

$\hat{B}={ }_{B} 0+\Delta B$

$n, t \quad n, t \quad n, t$

$t^{\prime}$--- necessary quantity of unknown parameters of element of $\hat{B}$;

$n, t$

$Y_{t^{\prime}, 1}^{0}=\left(Y_{1}^{0}, Y_{2}^{0}, \cdots, Y_{t^{\prime}}^{0}\right)^{T}$---approximations of $\hat{Y}_{t^{\prime}, 1}=\left(\hat{Y}_{1}, \hat{Y}_{2}, \cdots, \hat{Y}_{t^{\prime}}\right)^{T}$;

$\hat{y}=\left(\hat{y}_{1}, \hat{y}_{2}, \cdots, \hat{y}_{t^{\prime}}\right)^{T}$--- residuals of $Y_{t^{\prime}, 1}^{0}=\left(Y_{1}^{0}, Y_{2}^{0}, \cdots, Y_{t^{\prime}}^{0}\right)^{T}$;

$\hat{Y}_{t^{\prime}, 1}=Y_{t^{\prime}, 1}^{0}+\underset{t^{\prime}, 1}{\hat{y}}$

$X_{t, 1}^{0}=\left(X_{1}^{0}, X_{2}^{0} \cdots, X_{t}^{0}\right)^{T}$---approximations

of

$\hat{X}=\left(\hat{X}_{1,1}, \hat{X}_{2}, \cdots, \hat{X}_{t}\right)^{T}$;

$\hat{x}_{t, 1}=\left(\hat{x}_{1}, \hat{x}_{2}, \cdots, \hat{x}_{t}\right)^{T}$--residuals of $X_{t, 1}^{0}=\left(X_{1}^{0}, X_{2}^{0}, \cdots, X_{t}^{0}\right)^{T}$;

$\underset{t^{\prime}, 1}{\hat{X}}=X_{t^{\prime}, 1}^{0}+\hat{x}$

$A_{n, t^{\prime}}{ }^{---}$coming from $\underset{n, t^{\prime} t^{\prime}, 1}{A} \hat{Y}^{-} \underset{n, t}{\Delta} X_{t, 1}^{0 .}$

In Eq. 2, we don't consider $\Delta \hat{B} \hat{B} \hat{x}$, which is very small.

Obviously, Eq. 2 of this paper is different from homologous equations of literatures [1-3].

\subsection{Error Equations of Virtual Observations}

Because we suppose any element of $\hat{B}$ can be expressed
unknown parameters $\hat{Y}=\left(\hat{Y}_{1}, \hat{Y}_{2}, \cdots, \hat{Y}_{t^{\prime}}\right)^{T}, \quad$ and $\quad \hat{Y}_{i}$ and $\quad \hat{Y}_{j} \quad\left(1 \leq i, j \leq t^{\prime}\right.$ and $i \neq j$ ) are independent, so, if $\hat{B}_{i j}$ hasn't error,we don't list error equation;if $\hat{B}_{i j}$ has error, we list error equation

$V_{B_{i j}}=f_{i, t^{\prime}} \hat{y}_{t^{\prime}, 1}^{-}-\left(B_{i j}-B_{i j}^{0}\right), P_{B_{i j}}$

where:

$B_{i j}$--- observations of $\hat{B}_{i j}$;

$B_{i j}^{0}$--- approximations of $\hat{B}_{i j}$;

$V_{B_{i j}}{ }^{\text {--- residuals of }} B_{i j}$;

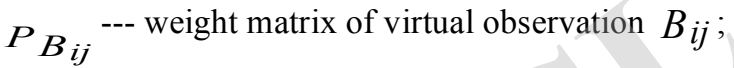

$f_{i j k}=\frac{\partial \hat{B}_{i j}}{\partial \hat{y}_{k}} \mid \hat{B}_{i j}=B_{i j}{ }^{0}, k=1,2, \cdots, t^{\prime} ;$

$f_{i, t^{\prime}}=\left(\begin{array}{llll}f_{i j 1} & f_{i j 2} & \cdots & f_{i j t^{\prime}}\end{array}\right)$.

Based on Eq.3, We can obtain

$$
V_{k, 1}=f_{k, t^{\prime} t^{\prime}, 1} \hat{y}_{k, 1} l_{B, k}, P_{k}
$$

where:

k---quantity of virtual observation $(0 \leq k \leq n t)$;

$P_{k, k}{ }^{---}$weight matrix of virtual observations;

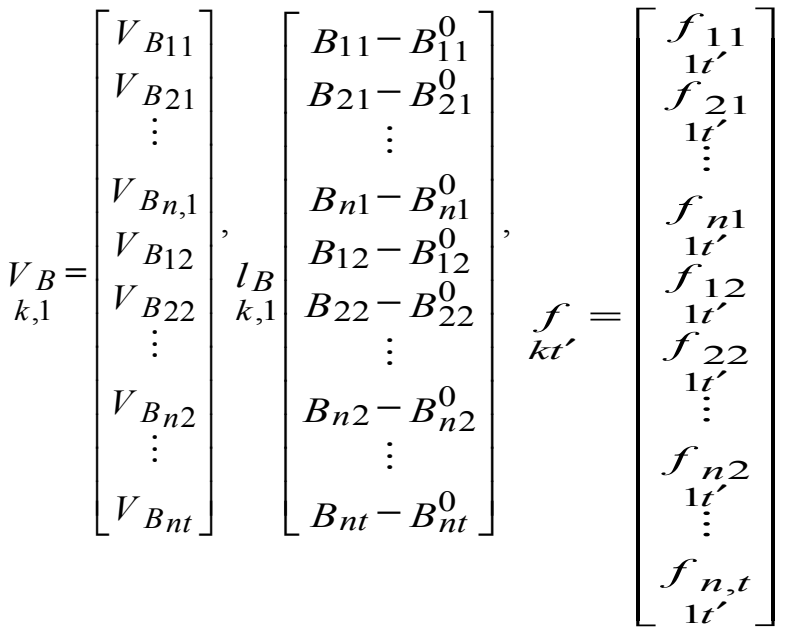

If $\hat{B}_{i j}$ hasn't error, we should delete homologous

elements of $V_{B}, f$ and $l_{B}$ in Eq.4 and homologous

$$
k, 1 \quad k, t^{\prime} \quad k, 1
$$

elements of

$$
P_{k, k}
$$

Obviously, Eq. 4 of this paper is still different from homologous equations of literatures [1-3]. 


\subsection{Total Error Equations}

Based on Eq. 2 and Eq. 4,we can obtain total error equations

$\underset{n+k, 1}{V}=\underset{n+k, t+t^{\prime}}{C} \underset{t+t^{\prime}, 1}{\hat{z}}-\underset{n+k, 1}{C}, P$

where:

$\underset{n+k, 1}{V}=\left[\begin{array}{c}V_{L} \\ n, 1 \\ V_{B} \\ k, 1\end{array}\right], \underset{n+k, t+t^{\prime}}{C}=\left[\begin{array}{cc}B^{0} & A \\ n, t & n, t^{\prime} \\ 0 & f \\ k, t & k, t^{\prime}\end{array}\right], \underset{t+t^{\prime}, 1}{\hat{z}}=\left[\begin{array}{c}\hat{x} \\ t, 1 \\ \hat{y} \\ t^{\prime}, 1\end{array}\right]$,

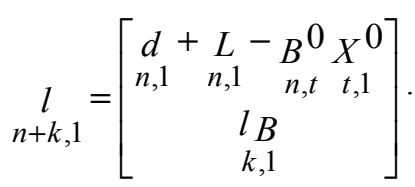

$P$--- total weight matrix;

$\underset{k, t}{0}$ of left bottom of $\left[\begin{array}{cc}B^{0} & A \\ n, t & n, t^{\prime} \\ 0 & f \\ k, t & k, t^{\prime}\end{array}\right]$ is zero matrix.

Obviously, Eq. 5 of this paper is different from homologous equations of literatures [1-3].

\subsection{Solving out Total Error Equations [4]}

Based on least square method and Eq. 5, we can obtain estimated values of unknown parameters

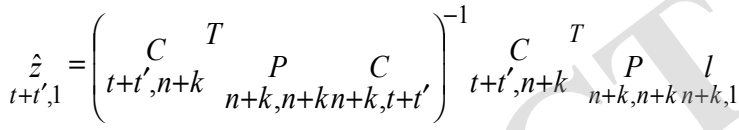

estimated value of mean square error of unit weight

$\hat{\sigma}_{0}= \pm \sqrt{\frac{V^{T} \stackrel{P}{1, n+k^{n+k, n+k} n+k, 1}}{(n+\mathrm{k})-\left(t+\mathrm{t}^{\prime}\right)}}$

and estimated values of variances and covariance of unknown parameters

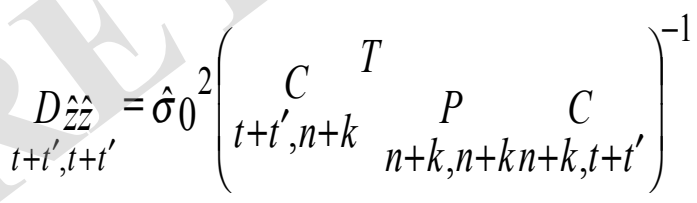

In this paper, $\hat{B}_{i j}$ may come from $\underset{t^{\prime}, 1}{\hat{Y}}$ or $V_{k}$, and results of the two method are not same, though the final alternate results are very small. In order to data consistence of estimated value of designing matrix, this paper selected that $\hat{B}_{i j}$ came from $\hat{Y}$. $t^{\prime}, 1$

Obviously, Eq. 6, Eq. 7 and Eq. 8 of this paper are different from homologous equations of literatures [1-3].

\section{FIRST IMITATIVE EXAMPLE}

Suppose there existed a plane curve such as

$\hat{Y}_{i}=\hat{a}_{0}+\hat{a}_{1} \hat{X}_{i}+\hat{a}_{2} \hat{X}_{i}^{2}+\hat{a}_{3} \hat{X}_{i}^{3} i=1,2, \cdots, n$

where:

$\hat{a}_{0}, \hat{a}_{1} \hat{a}_{2}$ and $\hat{a}_{3}{ }^{---}$estimated values of unknown coefficient;

$X_{i}$ and $Y_{i}{ }^{---}$measured data with error;

$\hat{X}_{i}$ and $\hat{Y}_{i}{ }^{---}$estimated value of $X_{i}$ and $Y_{i}$.

and we obtained coordinations of 10 points such as Table 1 based on imitative example.

Table 1. Measured data of plane curve.

\begin{tabular}{|c|c|c|}
\hline Point Name & $X(\mathbf{m})$ & $Y(\mathbf{m})$ \\
\hline \hline N0.1 & 3.536 & 125.145 \\
\hline N0.2 & 7.319 & 958.067 \\
\hline N0.3 & 1.527 & 15.860 \\
\hline N0.4 & 4.311 & 214.911 \\
\hline N0.5 & 5.473 & 418.513 \\
\hline N0.6 & 8.314 & 1384.036 \\
\hline N0.7 & 10.639 & 2834.032 \\
\hline N0.8 & 2.877 & 72.382 \\
\hline N0.9 & 13.521 & 5712.836 \\
\hline N0.10 & 15.392 & 8369.751 \\
\hline
\end{tabular}

\subsection{Error Equations of Actual Observations}

Based on Eq. 9, we can obtain error equation

$$
V_{10,1}=\hat{B}\left(\begin{array}{llll}
\hat{a}_{0} & \hat{a}_{1} & \hat{a}_{2} & \hat{a}_{3}
\end{array}\right)^{T}-\underset{10,1}{Y}, \underset{10 \Downarrow 10}{P_{Y}}
$$

where:

$$
\left.V Y=\left[\begin{array}{c}
V_{Y_{1}} \\
V_{Y_{2}} \\
\vdots \\
V_{Y_{10}}
\end{array}\right], \quad \hat{B}_{10,4}=\left[\begin{array}{cccc}
1 & \hat{X}_{1} & \hat{X}_{1}^{2} & \hat{X}_{1}^{3} \\
1 & \hat{X}_{2} & \hat{X}_{2}^{2} & \hat{X}_{2}^{3} \\
\vdots & \vdots & \vdots & \vdots \\
1 & \hat{X}_{n} & \hat{X}_{n}^{2} & \hat{X}_{n}^{3}
\end{array}\right], \quad \begin{array}{r}
Y \\
10,1
\end{array}\right]\left[\begin{array}{c}
Y_{1} \\
Y_{2} \\
\vdots \\
Y_{10}
\end{array}\right]
$$

$V_{Y_{i}}(i=1,2, \cdots, 10)$--- residuals of $Y_{i}(i=1,2, \cdots, 10)$;

PY --- weight matrix of actual observations.

Element of ${ }_{10,4} \hat{B}$ can be expressed by unknown parameters $\hat{X}_{10,1}=\left(\hat{X}_{1}, \hat{X}_{2}, \cdots, \hat{X}_{10}\right)^{T}$, and suppose $\hat{X}_{i}$ and $\hat{X}_{j} \quad(1 \leq i, j \leq 10$ and $i \neq j$ ) are independent.Then, we can obtain error equations of actual observations 
$\underset{10,1}{V_{Y}}=\left[\begin{array}{cc}B_{0} & A \\ 10,4 & 10,10\end{array}\right] \underset{14,1}{\hat{z}-l_{Y}}, \begin{gathered}P_{Y} \\ 10,1\end{gathered}$

where:

$B_{10,4}^{0}=\left[\begin{array}{cccc}1 & X_{1}^{0} & \left(X_{1}^{0}\right)^{2} & \left(X_{1}^{0}\right)^{3} \\ 1 & X_{2}^{0} & \left(X_{2}^{0}\right)^{2} & \left(X_{2}^{0}\right)^{3} \\ \vdots & \vdots & \vdots & \vdots \\ 1 & X_{10}^{0} & \left(X_{10}^{0}\right)^{2} & \left(X_{10}^{0}\right)^{3}\end{array}\right]$

$\underset{10,10}{A}=\operatorname{diag}\left(\begin{array}{llll}A_{1} & A_{2} & \cdots & A_{10}\end{array}\right)$

$\hat{z}=\left(\begin{array}{llllllll}\delta \hat{a}_{0} & \delta \hat{a}_{1} & \delta \hat{a}_{2} & \delta \hat{a}_{3} & \hat{x}_{1} & \hat{x}_{2} & \cdots & \hat{x}_{10}\end{array}\right)^{T} ;$

$\underset{10,1}{l_{Y}}=\left(\underset{10,1}{Y_{10,4}^{-}}{\underset{10}{0,1}}_{0}^{0} a^{0}\right)$

$X_{i}^{0}(i=1,2, \cdots, 10)$---approximations of $\hat{X}_{i}(i=1,2, \cdots, 10)$

$\hat{x}_{i}(i=1,2, \cdots, 10)$--- residuals of $X_{i}^{0}(i=1,2, \cdots, 10)$;

$\hat{X}_{i}=X_{i}^{0}+\hat{x}_{i}(i=1,2, \cdots, 10)$

$A_{i}=a_{1}{ }^{0}+2 a_{2}{ }^{0} X_{i}^{0}+3 a_{3}\left(X_{i}^{0}\right)^{2},(i=1,2, \cdots, 10)$

$a_{i}{ }^{0}(i=0,1,2,3)$---approximations of $\hat{a}_{i}(i=0,1,2,3)$;

$\delta \hat{a}_{i}(i=0,1,2,3)$--- residuals of $a_{i}^{0}(i=0,1,2,3)$;

$\hat{a}_{i}=a_{i}^{0}+\delta \hat{a}_{i}(i=0,1,2,3)$

$\underset{4,1}{a}=\left(\begin{array}{llll}0 & 0 & 0 & 0 \\ a_{0} & a_{1} & a_{2} & a_{3}\end{array}\right)^{T}$.

\subsection{Error Equations of Virtual Observations}

Because $\hat{B}_{i 1}=1(i=1,2, \cdots, 10)$ of $\hat{B}$ hasn't error,we 10,4

don't list error equation of virtual observation;and because others of $\hat{B}$ have error, we should list error equations of 10,4

virtual observations.

Because element of $\hat{B}$ can be expressed by parameters 10,4

$\hat{X}_{10,1}=\left(\hat{X}_{1}, \hat{X}_{2}, \cdots, \hat{X}_{10}\right)^{T}$, suppose $\hat{X}_{i}$ and $\hat{X}_{j} \quad(1 \leq i, j \leq 10$ and $i \neq j$ ) are independent,we can obtain error equations of virtual observations
$V_{B}=f \hat{x}-l_{B}, P_{B}$

$30,1 \quad 30,1010,1 \quad 30,1 \quad 30,30$

where:

$V_{30,1}=\left(\begin{array}{llllllllll}V_{B_{12}} & V_{B_{22}} & \cdots & V_{B_{102}} & V_{B_{13}} & V_{B_{23}} & \cdots & V_{B_{103}} & \cdots & V_{B_{104}}\end{array}\right)^{T}$

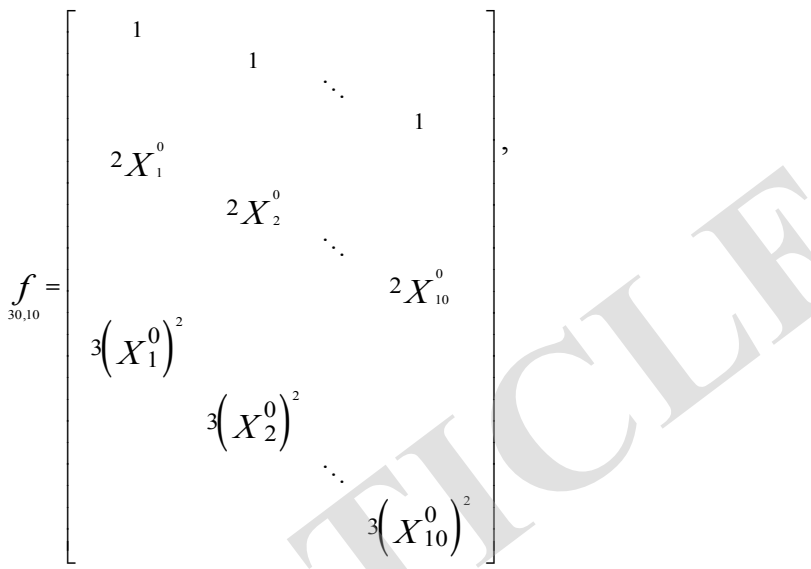

$\underset{10,1}{\hat{x}}=\left(\begin{array}{llll}\hat{x}_{1} & \hat{x}_{2} & \cdots & \hat{x}_{10}\end{array}\right)^{T}$,

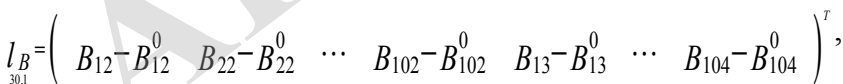

$\underset{30,30}{P}{ }^{---}$weight matrix of virtual observations.

\subsection{Total Error Equations}

Based on Eq. 11 and Eq. 12, we can obtain total error equations

$\underset{40,1}{V}=\underset{40,1414,1}{C} \hat{z}-l_{40,1}, \underset{40,40}{P}$

where:

$\underset{40,1}{V}=\left[\begin{array}{c}V_{Y} \\ 10,1 \\ V_{B} \\ 30,1\end{array}\right], \underset{40,14}{C}=\left[\begin{array}{cc}B^{0} & A \\ 10,4 & 10,10 \\ 0 & f \\ 30,4 & 30,10\end{array}\right], \underset{40,1}{l}=\left[\begin{array}{c}l_{Y} \\ 10,1 \\ l_{B} \\ 30,1\end{array}\right]$,

$\underset{30,4}{0}$ of left bottom of $\left[\begin{array}{cc}B^{0} & A \\ 10,4 & 10,10 \\ 0 & f \\ 30,4 & 30,10\end{array}\right]$ is zero matrix,

$P$--- total weight matrix.

40,40

\subsection{Solving out Total Error Equations}

Based on least square method and Eq.13, we can obtain estimated values of unknown parameters

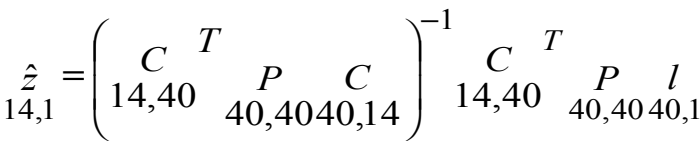

estimated value of mean square error of unit weight 
Table 2. Iterative Computing $\hat{a}_{0}, \hat{a}_{1}, \hat{a}_{2}$ and $\hat{a}_{3}$

\begin{tabular}{|c|c|c|c|c|}
\hline Iterative times & First & Second & Third & Fourth \\
\hline \hline$\hat{\sigma}_{0}$ & \pm 0.257497982 & \pm 0.25736601 & \pm 0.257366001 & \pm 0.257366001 \\
\hline$\hat{a}_{0}$ & -0.4282326 & -0.47119948 & -0.47160641 & -0.47161343 \\
\hline$\hat{a}_{1}$ & 2.91045167 & 2.92884547 & 2.929012661 & 2.929015863 \\
\hline$\hat{a}_{2}$ & 1.51054987 & 1.508330965 & 1.508311498 & 1.508311101 \\
\hline$\hat{a}_{3}$ & 2.18464297 & 2.1847223 & 2.184722976 & 2.184722991 \\
\hline
\end{tabular}

Table 3. Iterative computing variances and covariance.

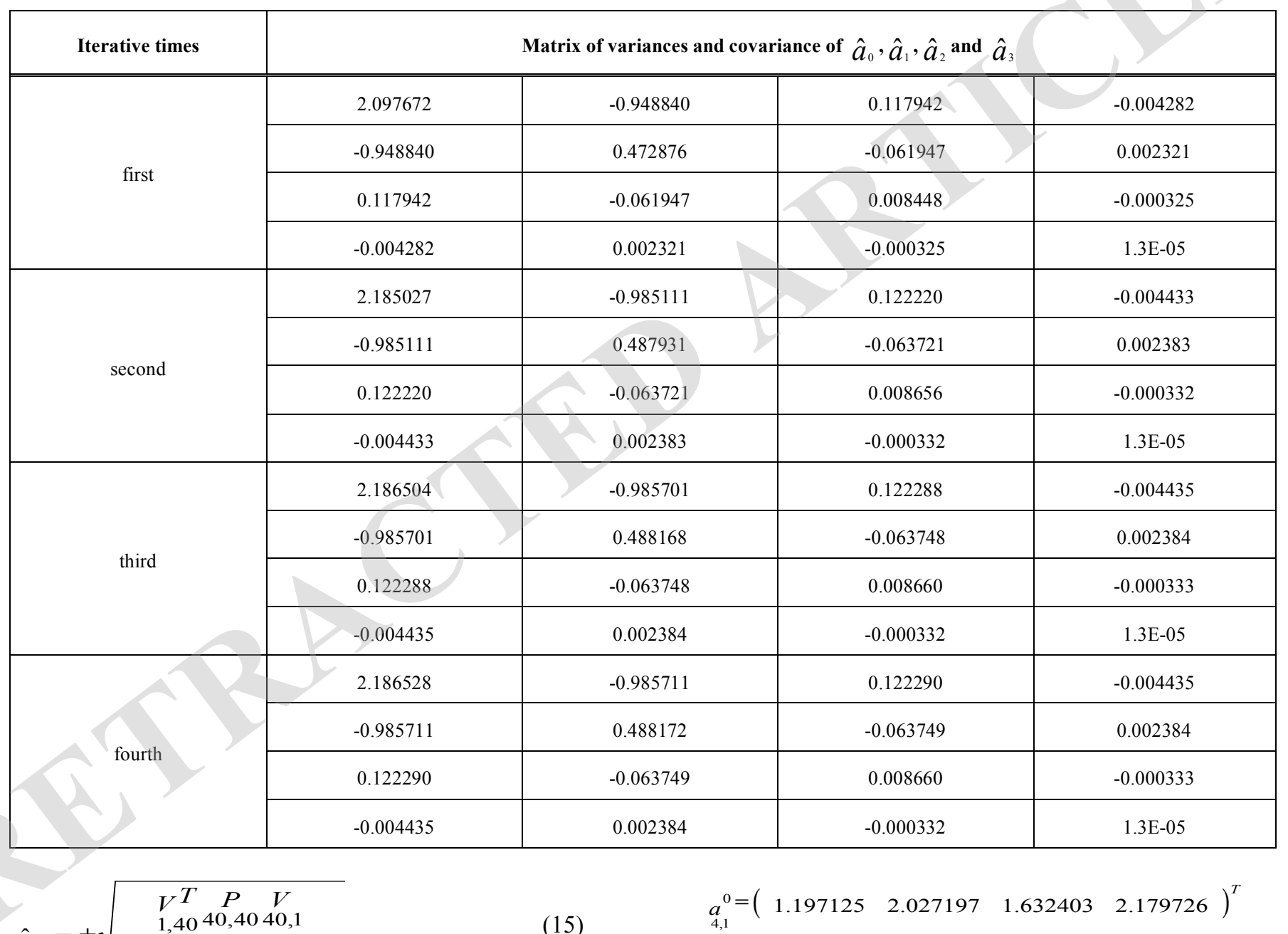

$\hat{\sigma}_{0}= \pm \sqrt{\frac{1,4040,4040,1}{(10+30)-(4+10)}}$

and estimated values of cofactor matrix of unknown parameters

$\underset{14,14}{D \hat{z} \hat{z}}=\hat{\sigma}_{0}^{2}\left(\begin{array}{ccc}C & T & \\ 14,40 & P & C \\ & 40,4040,14\end{array}\right)^{-1}$

\subsection{Results of Iterative Computing of This paper}

If we select No. 1, No. 2, No. 3 and No. 4 of Table 1, we can obtain approximations of unknown coefficient
In order to simplicity, this paper seleted total weight matrix $P$ as unit weight matrix, then, we can obtain Table 2 and 40,40

Table 3 according to new method of this paper,obtain Table 4 and Table 5 according to the method of Literatures [1-2], and obtain Table 6 and Table 7 according to Literatures [3].

Because our aim is to solve out curve, so, in Table 2, Table 3, Table 4, Table 5, Table 6, and Table 7, this paper only listed mean square error of unit weight,coefficients and their variances and covariance. 


\subsection{Results of Iterative Computing Based on Method of Literatures [1, 2]}

Table 4. Iterative Computing $\hat{a}_{0}, \hat{a}_{1}, \hat{a}_{2}$ and $\hat{a}_{3}$.

\begin{tabular}{|c|c|c|c|c|}
\hline Iterative times & First & Second & Third & Fourth \\
\hline \hline$\hat{\sigma}_{0}$ & \pm 0.377164774 & \pm 0.306303861 & \pm 0.293593374 & \pm 0.289064237 \\
\hline$\hat{a}_{0}$ & -0.08473767 & -2.40450675 & -3.29012507 & -3.98370589 \\
\hline$\hat{a}_{1}$ & 2.750551874 & 3.950898798 & 4.412274767 & 4.775093715 \\
\hline$\hat{a}_{2}$ & 1.530753891 & 1.370558058 & 1.308644367 & 1.259795641 \\
\hline$\hat{a}_{3}$ & 2.183902004 & 2.189966546 & 2.192319246 & 2.19417964 \\
\hline
\end{tabular}

Table 5. Iterative computing variances and covariance.

\begin{tabular}{|c|c|c|c|c|}
\hline Iterative times & \multicolumn{4}{|c|}{ Matrix of variances and covariance of $\hat{a}_{0}, \hat{a}_{1}, \hat{a}_{2}$ and $\hat{a}_{3}$} \\
\hline \multirow{3}{*}{ first } & 6.836696 & -3.205142 & 0.406789 & -0.014961 \\
\hline & 0.406789 & -0.223720 & 0.031304 & -0.001223 \\
\hline & -0.014961 & 0.008497 & -0.001223 & $4.9 \mathrm{E}-05$ \\
\hline \multirow{3}{*}{ second } & -2.181921 & 1.137142 & -0.152644 & 0.005802 \\
\hline & 0.273889 & -0.152644 & 0.021566 & -0.000848 \\
\hline & -0.010007 & 0.005802 & -0.000848 & 3.4E-05 \\
\hline third & -0.020657 & 0.011539 & -0.001640 & $6.5 \mathrm{E}-05$ \\
\hline \multirow{4}{*}{ fourth } & 10.780828 & -5.159566 & 0.662257 & -0.024521 \\
\hline & -5.159566 & 2.683191 & -0.359551 & 0.013649 \\
\hline & 0.662257 & -0.359551 & 0.049830 & -0.001935 \\
\hline & -0.024521 & 0.013649 & -0.001935 & $7.6 \mathrm{E}-05$ \\
\hline
\end{tabular}

3.7. Results of Iterative Computing Based on Method of Literatures [3]

Table 6. Iterative Computing $\hat{a}_{0}, \hat{a}_{1}, \hat{a}_{2}$ and $\hat{a}_{3}$.

\begin{tabular}{|c|c|c|c|c|}
\hline Iterative times & First & Second & Third & Fourth \\
\hline \hline$\hat{\sigma}_{0}$ & \pm 0.257497982 & \pm 0.25736601 & \pm 0.257366001 & \pm 0.257366001 \\
\hline$\hat{a}_{0}$ & -0.4282326 & -0.47119948 & -0.47160641 & -0.47161343 \\
\hline$\hat{a}_{1}$ & 2.91045167 & 2.92884547 & 2.929012661 & 2.929015863 \\
\hline$\hat{a}_{2}$ & 1.51054987 & 1.508330965 & 1.508311498 & 1.508311101 \\
\hline$\hat{a}_{3}$ & 2.18464297 & 2.1847223 & 2.184722976 & 2.184722991 \\
\hline
\end{tabular}


Table 7. Iterative computing variances and covariance.

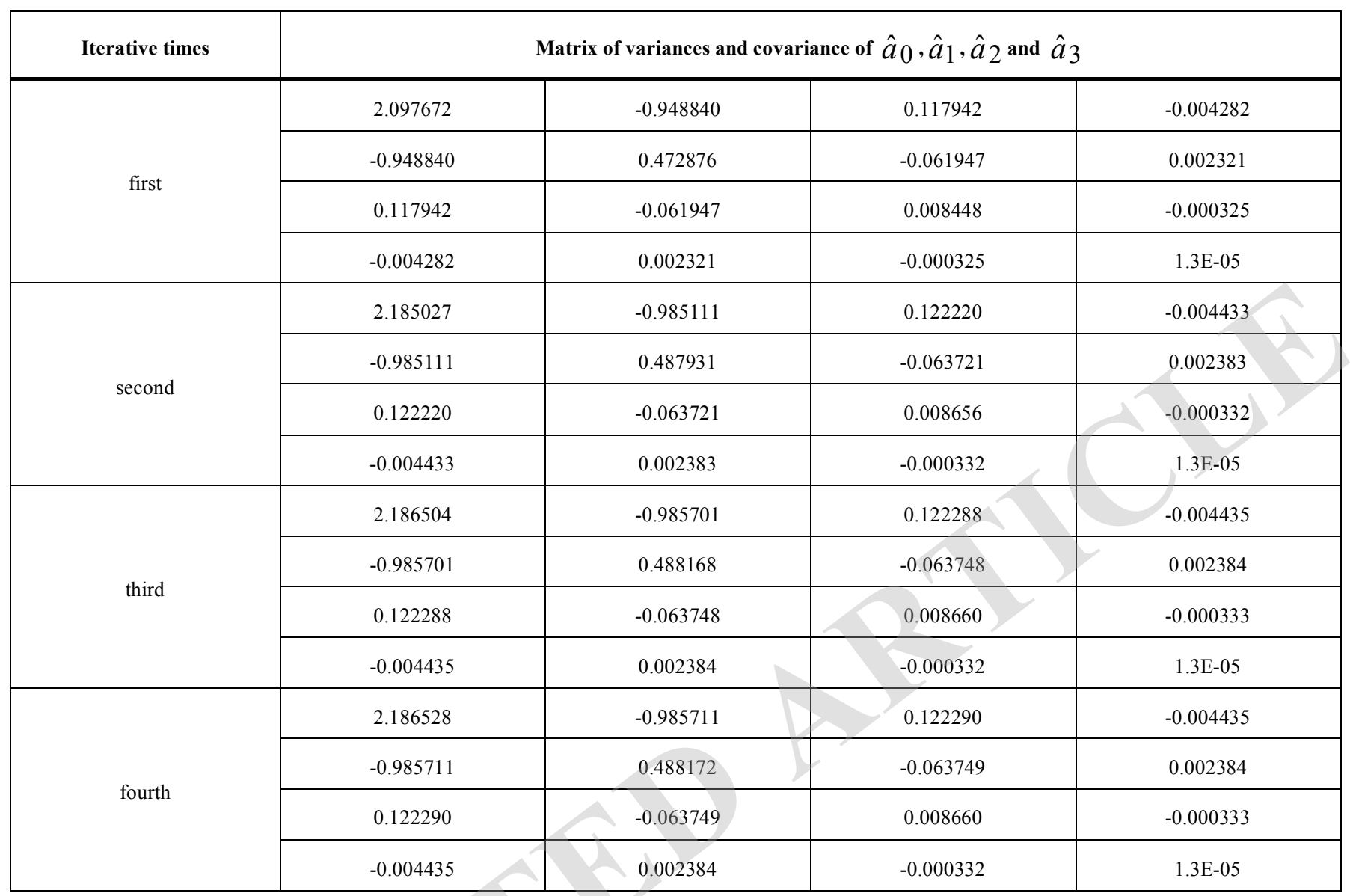

\subsection{Results of Comparison Analysis}

From Table 2, Table 3, Table 4, Table 5, Table 6, and Table 7 of this imitative example, we can find out:

(1) New method of this paper is better at algorithm being stable, mean square error of unit weight being small, and matrix of variances and covariance of $\hat{a}_{0}, \hat{a}_{1}, \hat{a}_{2}$ and $\hat{a}_{3}$ being stable and small than the method of literatures $[1,2]$.

(2) The results of new method of this paper is almost as same as the results of the method of literature [3].

In a word, this example showed that new mothod of this paper is viable and valid and still supported literature [3].

\section{SECOND IMITATIVE EXAMPLE}

If Table $\mathbf{1}$ changes into Table $\mathbf{8}$, we can obtain Table $\mathbf{9}$ and Table $\mathbf{1 0}$ according to new method of this paper,obtain Table 11 and Table $\mathbf{1 2}$ according to the method of Literatures [1-2], and obtain Table 13 and Table 14 according to Literatures [3].

If we select No. 1, No. 2, No. 3 and No. 4 of Table 8, we can obtain approximations of unknown coefficient $\underset{4,1}{a}=\left(\begin{array}{llll}2.360120 & 1.788430 & 0.341557 & 0.725756\end{array}\right)^{T}, \quad$ and obtain follow computation.

\subsection{Results of Iterative Computing of This paper}

Table 8. Measured data of plane curve.

\begin{tabular}{|c|c|c|}
\hline Point name & $X$ (m) & $Y$ (m) \\
\hline \hline N0.1 & 1.363 & 7.270 \\
\hline N0.2 & 0.596 & 3.701 \\
\hline N0.3 & 2.419 & 18.958 \\
\hline N0.4 & 1.541 & 8.583 \\
\hline N0.5 & 3.125 & 33.061 \\
\hline N0.6 & 2.113 & 14.587 \\
\hline N0.7 & 1.894 & 11.981 \\
\hline N0.8 & 2.151 & 15.084 \\
\hline N0.9 & 0.953 & 4.962 \\
\hline N0.10 & 1.581 & 8.951 \\
\hline
\end{tabular}


Table 9. Iterative computing $\hat{a}_{0}, \hat{a}_{1}, \hat{a}_{2}$ and $\hat{a}_{3}$.

\begin{tabular}{|c|c|c|c|c|}
\hline Iterative times & First & Second & Third & Fourth \\
\hline \hline$\hat{\sigma}_{0}$ & \pm 0.006406091 & \pm 0.006406885 & \pm 0.006406885 & \pm 0.006406885 \\
\hline$\hat{a}_{0}$ & 2.61877099 & 2.61971445 & 2.61972163 & 2.61972169 \\
\hline$\hat{a}_{1}$ & 1.03091098 & 1.03000236 & 1.02998864 & 1.02998853 \\
\hline$\hat{a}_{2}$ & 0.94419441 & 0.94439666 & 0.94440431 & 0.94440437 \\
\hline$\hat{a}_{3}$ & 0.58970185 & 0.58970223 & 0.58970094 & 0.58970094 \\
\hline
\end{tabular}

Table 10. Iterative computing variances and covariance.

\begin{tabular}{|c|c|c|c|c|}
\hline \multirow{2}{*}{ first } & 0.001725 & -0.003180 & 0.001738 & -0.000288 \\
\hline & 0.001738 & -0.003497 & 0.002018 & -0.000348 \\
\hline \multirow{3}{*}{ second } & -0.003069 & 0.006029 & -0.003416 & 0.000580 \\
\hline & 0.001683 & -0.003416 & 0.001979 & -0.000341 \\
\hline & -0.000280 & 0.000580 & -0.000341 & 0.000060 \\
\hline third & -0.000280 & 0.000580 & -0.000341 & 0.000060 \\
\hline \multirow{4}{*}{ fourth } & 0.001650 & -0.003068 & 0.001683 & -0.000280 \\
\hline & -0.003068 & 0.006026 & -0.003414 & 0.000580 \\
\hline & 0.001683 & -0.003414 & 0.001978 & -0.000341 \\
\hline & -0.000280 & 0.000580 & -0.000341 & 0.000060 \\
\hline
\end{tabular}

4.2. Results of Iterative Computing Based on Method of Literatures [1-2]

Table 11. Iterative computing $\hat{a}_{0}, \hat{a}_{1}, \hat{a}_{2}$ and $\hat{a}_{3}$.

\begin{tabular}{|c|c|c|c|c|}
\hline Iterative times & First & Second & Third & Fourth \\
\hline \hline$\hat{\sigma}_{0}$ & \pm 0.008408085 & \pm 0.010151230 & \pm 0.010207209 & \pm 0.010210152 \\
\hline$\hat{a}_{0}$ & 2.63276735 & 2.61192737 & 2.62314999 & 2.62202865 \\
\hline$\hat{a}_{1}$ & 1.00400443 & 1.04681364 & 1.02324480 & 1.02557058 \\
\hline$\hat{a}_{2}$ & 0.95924510 & 0.93430879 & 0.94825276 & 0.94688871 \\
\hline$\hat{a}_{3}$ & 0.58716728 & 0.59148182 & 0.58904186 & 0.58927905 \\
\hline
\end{tabular}


Table 12. Iterative computing variances and covariance.

\begin{tabular}{|c|c|c|c|c|}
\hline \multirow{3}{*}{ first } & 0.005260 & -0.010251 & 0.005794 & -0.000984 \\
\hline & 0.005794 & -0.012296 & 0.007328 & -0.001289 \\
\hline & -0.000984 & 0.002129 & -0.001289 & 0.000230 \\
\hline \multirow{3}{*}{ second } & -0.009952 & 0.020474 & -0.011937 & 0.002067 \\
\hline & 0.005623 & -0.011937 & 0.007116 & -0.001252 \\
\hline & -0.000954 & 0.002067 & -0.001252 & 0.000223 \\
\hline third & -0.001004 & 0.002172 & -0.001315 & 0.000234 \\
\hline \multirow{4}{*}{ fourth } & 0.005291 & -0.010312 & 0.005829 & -0.000989 \\
\hline & -0.010312 & 0.021213 & -0.012367 & 0.002141 \\
\hline & 0.005829 & -0.012367 & 0.007369 & -0.001296 \\
\hline & -0.000989 & 0.002141 & -0.001296 & 0.000231 \\
\hline
\end{tabular}

\subsection{Results of Iterative Computing Based on Method of Literatures [3]}

Table 13. Iterative computing $\hat{a}_{0}, \hat{a}_{1}, \hat{a}_{2}$ and $\hat{a}_{3}$.

\begin{tabular}{|c|c|c|c|c|}
\hline Iterative times & First & Second & Third & Fourth \\
\hline \hline$\hat{\sigma}_{0}$ & \pm 0.00640609 & \pm 0.006406885 & \pm 0.006406885 & \pm 0.006406885 \\
\hline$\hat{a}_{0}$ & 2.61877099 & 2.61971445 & 2.61972163 & 2.61972169 \\
\hline$\hat{a}_{1}$ & 1.03091098 & 1.03000236 & 1.02998864 & 1.02998853 \\
\hline$\hat{a}_{2}$ & 0.94419441 & 0.94439666 & 0.94440431 & 0.94440437 \\
\hline$\hat{a}_{3}$ & 0.58970185 & 0.58970223 & 0.58970094 & 0.58970094 \\
\hline
\end{tabular}

Table 14. Iterative computing variances and covariance.

\begin{tabular}{|c|c|c|c|c|}
\hline \multirow{3}{*}{ Iterative times } & \multicolumn{4}{|c|}{ Matrix of variances and covariance of $\hat{a}_{0}, \hat{a}_{1}, \hat{a}_{2}$ and $\hat{a}_{3}$} \\
\hline \hline \multirow{3}{*}{ first } & 0.001725 & -0.003180 & 0.001738 & -0.000288 \\
\cline { 2 - 5 } & -0.003181 & 0.006195 & -0.003497 & 0.000593 \\
\cline { 2 - 5 } & 0.001738 & -0.003497 & 0.002018 & -0.000348 \\
\cline { 2 - 5 } & -0.000288 & 0.000593 & -0.000348 & 0.000061 \\
\cline { 2 - 5 } & 0.001651 & -0.003069 & -0.001683 & -0.000280 \\
\cline { 2 - 5 } & -0.003069 & 0.006029 & 0.001979 & 0.000580 \\
\cline { 2 - 5 } & 0.001683 & -0.003416 & -0.000341 & -0.000341 \\
\hline
\end{tabular}


(Table 14) contd.....

\begin{tabular}{|c|c|c|c|c|}
\hline \multirow{3}{*}{ Iterative times } & \multicolumn{4}{|c|}{ Matrix of variances and covariance of $\hat{a}_{0}, \hat{a}_{1}, \hat{a}_{2}$ and $\hat{a}_{3}$} \\
\hline \hline \multirow{3}{*}{ third } & 0.001650 & -0.003068 & 0.001683 & -0.000280 \\
\cline { 2 - 5 } & -0.003068 & 0.006026 & -0.003414 & 0.000580 \\
\cline { 2 - 5 } & 0.001683 & -0.003414 & 0.001978 & -0.000341 \\
\cline { 2 - 5 } & -0.000280 & 0.000580 & -0.000341 & 0.000060 \\
\hline \multirow{3}{*}{ fourth } & 0.001650 & -0.003068 & 0.001683 & -0.000280 \\
\cline { 2 - 5 } & -0.003068 & 0.006026 & -0.003414 & 0.000580 \\
\cline { 2 - 5 } & 0.001683 & -0.003414 & -0.001978 & -0.000341 \\
\cline { 2 - 5 } & -0.000280 & 0.000580 & 0.000341 & 0 \\
\hline
\end{tabular}

\subsection{Results of Comparison Analysis}

From Table 9, Table 10, Table 11, Table 12, Table 13, and Table 14 of second imitative example, we can obtain same results as that of first imitative example.

\section{CONCLUSION}

The new method of this paper is more strict in theory, because it thinks elements of designing matrix may have condition equations, and selects essential unknown parameters to express all virtual observation values among designing matrix.

The new method of this paper is another improved method which isn't the same as the method of literature [3], and has been deduced. So, this paper perfected and enriched total least square algorithm in theoretics.

Two examples showed that new mothod of this paper is viable and valid, and still supported and perfected literature [3] in practice.

\section{CONFLICT OF INTEREST}

The authors confirm that this article content has no conflict of interest.

\section{ACKNOWLEDGEMENTS}

Declared none.

\section{REFERENCES}

[1] E. H. Wei, Z. X, Yin, G. W. Li, and Z. Q. Li, "On 3D coordinate transformations with virtual observation method," Geomatics and Information Science of Wuhan University, vol. 39, no. 2, pp. 152156, 2014.

[2] Y. B. Yao, and J. Kong, "A new combined ls method considering random errors of design matrix," Geomatics and Information Science of Wuhan University, vol. 39, no. 9, pp. 1028-1032, 2014.

[3] Y.H. Deng, "Improved total least square algorithm", The Open Civil Engineering Journal, vol. 9, pp. 394-399, 2015.

[4] Teaching and Research Group of Surveying Adjustment, Error Theory and Fundation of Surveying Adjustment $2^{\text {nd }}$ ed., School of Geodesy and Geomatics of Wuhan University, CA: Wuhan, pp. 106-128, 2003.

(C) Deng Yonghe; Licensee Bentham Open.

This is an open access article licensed under the terms of the (https://creativecommons.org/licenses/by/4.0/legalcode), which permits unrestricted, noncommercial use, distribution and reproduction in any medium, provided the work is properly cited. 